\title{
Erratum
}

\section{Minimum Norm Quadratic Estimation of Components of Spatial Covariance}

The following corrections should be made in our recent paper (Marshall and Mardia, 1985):

p. $520, \ell 7$ delete the sentence, "Further ..."

p. $522, \ell 15$ Multiply the R.H.S. of $\operatorname{var}\left(\bar{\sigma}_{1}^{2}\right)$ by 2 .

$\ell 16$ In the R.H.S. of var $\left(\sigma_{1}^{2}\right)$, replace $\left(\frac{1+\rho^{2}}{1-\rho^{2}}\right)$ by

$$
\left(\frac{1+\rho^{2}}{1-\rho^{2}}\right)^{3} \text {. }
$$

Multiply the R.H.S. of $\operatorname{var}\left(\bar{\sigma}_{2}^{2}\right)$ by 2 .

$\ell 18$ Delete the sentence "It is not . . ."

Table 1 In the row for $\sigma_{1}^{2}, \mathrm{M} 2$, replace the entries by $43.5,32.2$, $21.8,11.3$, respectively.

In the row for $\sigma_{1}^{2}$, M3, replace ' 85.6 ' by ' 86.0 '.

In $\sigma_{2}^{2}$, replace the entries by $42.5,25.2,9.6,1.4$, respectively.

\section{REFERENCE}

Marshall, R. J. and Mardia, K. V., 1985, Minimum norm quadratic estimation of components of spatial covariance: Math. Geol, v. 17, no. 5, p. 517-525.

K. V. Mardia

Department of Statistics

University of Leeds

Leeds LS2 9JT England 